\title{
LOS VERBOS DE ASCENSO COMO EXPRESIONES MODALES: EL CASO DEL ESPAÑOL
}

\author{
DIEGO GABRIEL KRIVOCHEN \\ Universidad Nacional de La Plata \\ diegokrivochen@hotmail.com
}

\section{RESUMEN}

En este trabajo reanalizaremos una estructura sintáctica que ha sido objeto de estudio en la Gramática Generativa desde la década del los 70 (por ejemplo, Chomsky 1973, Postal 1974): las estructuras de "ascenso". En estas estructuras hay un verbo que selecciona una cláusula subordinada no finita, en la que un argumento recibe rol temático pero no caso, produciéndose un movimiento-A hacia la cláusula matriz para recibir caso. Este sistema implica grandes complicaciones a nivel teórico y empírico, por lo que intentaremos reemplazar esa teoría por la de que los así llamados "verbos de ascenso" (parecer como ejemplo prototípico) son en realidad auxiliares con un significado modal. Así, no hay dos cláusulas, sino sólo una, con un dictum y un modus, en la que el verbo de ascenso es en realidad un núcleo con rasgos modales. Comenzaremos caracterizando la noción de modalidad en diferentes ámbitos y luego nos centraremos en el estudio de la sintaxis y la semántica de los verbos de ascenso desde la convergencia entre generativismo, Relevancia y conceptos de lógica modal.

PALABRAS CLAVE: ascenso; modalidad; interfaz sintaxis-semántica.

\section{RAISING VERBS AS MODAL EXPRESSIONS: THE CASE OF SPANISH}

\section{ABSTRACT}

In this paper we will reanalyze a syntactic structure that has been studied in Generative Grammar since the '70s (e.g., Chomsky 1973, Postal 1974): "raising" structures. In these structures there is a verb that selects a non-finite subordinate clause in which an argument receives a thematic role but no case, resulting in a movement-A to the matrix clause for case checking reasons. This system involves major complications at both the theoretical and empirical levels, so we will try to replace that theory for the claim that so-called "raising verbs" are actually auxiliaries conveying modal meaning. Thus, there are not two clauses, but only one with a dictum and a modus, in which the raising verb is a modal head. We will begin by characterizing the notion of modality in different areas and then we will focus on the study of syntax and semantics of raising verbs from the convergence of generativism, Relevance Theory and concepts from modal logics.

KEY WORDS: raising; modality; syntax-semantics interface.

\section{Modalidad}

La modalidad es, a grandes rasgos, la expresión lingüística de la subjetividad del hablante respecto del contenido proposicional. Así, podemos considerar en una primera instancia que la modalidad puede expresarse en términos de una función $f(x)$, en la que $f$ es la Modalidad, considerada en términos amplios (ya veremos por qué) y $x$ es el contenido proposicional. No obstante, tenemos que 
tener en cuenta que la modalidad se presenta como una relación compleja y múltiple, entre el locutor y su enunciado, y entre el locutor y el alocutario, por lo que excedería, aparentemente, el ámbito del estudio gramatical. Intentaremos demostrar que no es así.

García Negroni y Tordesillas Colado (2001) hacen una distinción que nos parece útil como introducción al tema: Modalidad Lingüística por un lado y Modalidad Lógica por el otro. Ampliaremos esta distinción desde el punto de vista de la lingüística discursiva, para luego fundamentar nuestra hipótesis de que la sintaxis pre-sub-determina la inferencia y el discurso, en el sentido en que no hay nada en el discurso que no esté previsto por una categoría gramatical, así como los procesos discursivos pueden, a su vez, efectuar cambios en el output de la sintaxis si este no lleva a la relevancia óptima. El objetivo es un esbozo de integracionismo dentro de los límites de una teoría científica, como venimos sosteniendo desde Krivochen (2011b).

\subsection{Modalidad lingüística}

Dentro de los estudios lingüísticos, debemos mencionar las teorías de Charles Bally y Emile Benveniste, en orden cronológico, como las principales, y las que servirán de apoyo a nuestro planteo. Las formulaciones serán reseñadas en términos contemporáneos, de modo tal que sean útiles tanto para el analista del discurso como para el sintactista.

Según Bally (1932), la distinción fundamental que debe hacerse es aquella entre el contenido proposicional y el punto de vista del hablante respecto de ese contenido proposicional, el cual puede expresarse mediante variados recursos lingüísticos (sintácticos, prosódicos y semánticos). La definición de Bally es la siguiente (1932: 35): "modalidad es la forma lingüística de un juicio intelectual, de un juicio afectivo o de una voluntad o de una representación de su espíritu". Así, distingue modalidad intelectual, afectiva y volitiva. Procederemos a ejemplificar:

Tomemos el siguiente contenido proposicional, expresado en términos de una función $f(x)$ (obviando el adjunto, ya que su status es conflictivo desde las teorías sintácticas actuales y no es pertinente a nuestra argumentación):

(1) Venir (Juan)

A este mismo contenido proposicional pueden modificarlo diferentes indicadores modales, sin que haya cambios en términos lógico-veritativos, ya que estos afectan a la proposición, no al functor proposicional:

Relación modal intelectual: formulada en términos de grado de certeza respecto del contenido proposicional

a) Juan viene hoy

b) Es posible que Juan venga hoy 
Relación modal afectiva: en términos de evaluación apreciativa del hablante como bueno-malo

a) Por suerte, Juan viene hoy

b) Es lamentable que Juan venga hoy

Relación modal volitiva: en términos del deseo del hablante de que el contenido proposicional sea el caso

a) ¡Ojalá Juan venga hoy!

b) No quiero que Juan venga hoy

Normalmente, estos indicadores modales se encuentran en distribución complementaria: no suele ser el caso que el mismo sujeto modal haga, por ejemplo, un juicio volitivo y afectivo y la oración resultante sea aceptable sin una considerable carga de inferencias no automáticas; aunque es posible combinar la relación intelectual con las otras dos, ${ }^{1}$ como en los siguientes ejemplos:

(2) Por suerte, es verdad que Juan viene hoy

(3) ¡Ojalá sea cierto que Juan viene hoy!

Hablamos de modalidad explícita cuando el indicador modal está materializado, de lo contrario, hablaremos de modalidad implícita. Sea implícita o explícita, la modalidad (o, mejor dicho, el modus) está compuesto de un sujeto modal, aquel que realiza el juicio, y un verbo modal, que explicita el tipo de reacción / juicio respecto del contenido proposicional. Así, la estructura modal de una oración será siempre: ${ }^{2}$

(4) [Modus sujeto modal + verbo modal [Dictum predicación (referencia)]]

Benveniste (1977) introduce el concepto de modalidad en relación a la noción de enunciación. Define a esta última como "la puesta en funcionamiento de la lengua por un acto individual de utilización", acto que define al "hablante" como la persona subjetiva $\left(1 .{ }^{\mathrm{ra}}\right)$, alrededor de y en oposición a la cual

1 Aunque no será investigada aquí, existe la posibilidad de proponer, al modo cartográfico (siguiendo y ampliando las propuestas de Cinque 1999, 2006) una proyección modal para cada tipo de modalidad, de modo que no haya conflicto en la co-ocurrencia de dos elementos modales de diferente valor semántico. El resultado sería una "Split-ModP", en consonancia con las propuestas sobre Split-PP (ver los artículos en Cinque y Rizzi 2010).

2 La propuesta puede parecer reminiscente de la llamada "Hipótesis realizativa", ya que ambas intentan explicar la estructura de una oración performativa (los enunciados modales son actos expresivos en términos de Searle 1975) en la que el indicador no está materializado a partir de una estructura lógica subyacente en la que el indicador aparece. Nos remitimos, entonces, a los primeros modelos generativos, los verdaderamente "transformacionales". 
se definen la persona no-subjetiva (2. ${ }^{\mathrm{da}}$ ) y la no-persona (3. $\left.{ }^{\mathrm{ra}}\right)$. En la enunciación, el sujeto se apropia del aparato formal de la lengua, que consiste de:

\section{a) Deícticos}

b) Procedimientos accesorios

Estos últimos establecen las características de la relación hablante-oyente por un lado, y hablante-enunciado por el otro. Estas relaciones se establecen haciendo uso de elementos que se encuentran sintácticamente fuera de la proposición y que están relacionados con el concepto de fuerza ilocucionaria (Austin 1962), licenciada por elementos ubicados en la llamada "periferia izquierda" (Rizzi 1997, 2004), que nosotros revisaremos más adelante:

a) La aserción (caracterizada a veces como el "grado cero" de la modalidad)

b) La interrogación

c) La intimación

Las dos últimas implican una acción del alocutario, mientras que la primera se caracteriza por describir un estado de cosas. La interrogación, además, es la más compleja en términos sintácticos, ya que normalmente requiere, aparte de una entonación específica, un movimiento de constituyentes (excepto en las llamadas "echo questions", en las que el Qu- permanece in situ) a la periferia izquierda (el dominio del Sintagma Complementante), un Qu- al especificador y el verbo a $\mathrm{C}_{0}$ para dar el orden final [с $\mathrm{Qu}-\mathrm{j}\left[\mathrm{Q}+\left(\mathrm{V}+\mathrm{T}_{\mathrm{i}}\right){ }_{\text {т }} \mathrm{Suj}\left[\mathrm{h}_{\mathrm{i}}{ }_{v}\right.\right.$ $\left.h_{\mathrm{j}}\right]$ ]]l] (obviando el movimiento de $\mathrm{V}$ a $\mathrm{T}$, el del sujeto de especificador de $v$ a especificador de $\mathrm{T}$, etc.).

Desde una perspectiva también enunciativa, Maigueneau (1980), por su parte, describe tres tipos de modalidad, que se manifiestan mediante diferentes estrategias léxico-sintácticas:

a) Modalidad de enunciado: corresponde a la evaluación en términos epistémicos o deónticos, es decir, certeza respecto del contenido proposicional o evaluación del mismo en términos de bueno-malo.

b) Modalidad de enunciación: corresponden a los procedimientos accesorios de Benveniste, aunque tanto Maingueneau como García Negroni y Tordesillas Colado incluyen elementos modales tales como adverbios (también podríamos incluir adjetivos, sustantivos) generados en la periferia izquierda, en posición de especificador de STóp. El concepto de modalidad de enunciación vincula el tema de la modalidad con la teoría de los Actos de Habla (Austin, Searle) y pone a la oración en contexto, en relación a un locutor y a un alocutario. 
c) Modalidad de mensaje: son transformaciones gramaticales con valor modalizador. Dentro de estas podemos ubicar a la pasiva (con y sin agente realizado léxicamente), la ergativización de una estructura transitiva mediante el clítico -se, entre otras. Pese a que en la gramática transformacional (estrictamente, la gramática generativa hasta mediados de la década de los 70) se establece una relación derivacional entre, por ejemplo, la activa y la pasiva, o la construcción de objeto indirecto preposicional y la construcción de doble objeto (Larson 1988), nosotros consideramos que cada una de esas construcciones son base-generated con fundamentos en los requerimientos de interfaz (Krivochen y Kosta 2013).

La sección siguiente presentará un enfoque lógico respecto de la modalidad, en términos de lógica veritativa-proposicional, siempre en estrecha relación con planteos lingüísticos.

\subsection{Lógica Modal}

Dentro de la lógica contemporánea, hay toda una rama que se dedica al estudio de la modalidad, con una perspectiva relacionada, pero diferente a la Lingüística. Mientras que para la Lingüística (discursivamente orientada) es fundamental la relación del hablante con el contenido proposicional, y la modalidad es la evaluación del hablante respecto de este contenido en términos más o menos explícitamente subjetivos, para la Lógica lo fundamental es el concepto (primitivo, y raramente definido) de verdad. Para la lógica modal, entonces, las modalidades fundamentales son aquellas que se relacionan con la verdad del contenido de las proposiciones. Así, un juicio (la unidad de análisis para la lógica modal) puede presentarse como un hecho (juicio categórico), una posibilidad (juicio hipotético) o una necesidad (juicio apodíctico). Estas tres dimensiones definen el primer gran tipo de modalidad lógica, la llamada alética. Esta subclasificación incluye las categorías de lo posible, lo imposible, lo necesario y lo contingente, obteniendo así una taxonomía rica en matices lógicos, aunque no siempre distinguibles claramente en el dominio del lenguaje natural. Analizaremos un ejemplo más abajo.

Dentro de la lógica, el concepto de modalidad se extiende también a otros dominios, ya conocidos: la certeza y el deber. Hablamos así de modalidades lógicas epistémicas, que incluyen a lo seguro, lo dudoso, lo excluido y lo probable; y las deónticas, que incluyen a lo obligatorio, lo permitido, lo prohibido y lo facultativo. Caracterizaciones de la modalidad tales como Palmer (1986) resultan especialmente compatibles con este planteo, aunque debemos tener en cuenta que la habilidad (lo facultativo) suele ser incluido dentro de la modalidad epistémica en la literatura lingüística sobre el tema. Veamos ahora un ejemplo simple para analizar cómo las distinciones lógicas no siempre son claras en el ámbito del lenguaje natural:

(5) Es necesario que veas a Juan hoy 
Podemos sentirnos inclinados a decir que hay necesidad en sentido lógico estricto (es decir, $p$ es verdadera a priori y analíticamente) por la presencia del elemento léxico explícito, pero esa necesidad es en realidad a posteriori (sintéticamente) y deóntica a pesar de lo que las piezas fonológicas sugieren: el significado de (5) puede parafrasearse como "debes ver a Juan hoy". ${ }^{3}$ Lo que sostenemos es que la operación de Inserción de Vocabulario (Spell-Out, o "materialización") puede aplicarse a diferentes estructuras lógicas y generar como resultado la misma forma fonológica, originando así ambigüedades y probando que la interfaz sintaxis-fonología no es unívoca, no obstante manteniéndose la información semántica para la transferencia al nivel de interfaz Forma Lógica (ver, por ejemplo, Huang 1995, Hornstein 1995 para análisis del nivel de Forma Lógica y las operaciones que tienen lugar en él, involucrando principalmente fenómenos de alcance con negación y cuantificación). En términos más generales, ninguna interfaz cognitiva es transparente, en el sentido en que el mapeo siempre implica alguna transformación de información, aunque nunca pérdida o creación (respetando así el segundo principio de la Termodinámica, o Principio de Conservación). Lo que acabamos de decir puede graficarse del siguiente modo, aplicando el razonamiento a la negación (Peter Kosta, p.c. confirma que en ruso las tres opciones pueden compartir materialización):

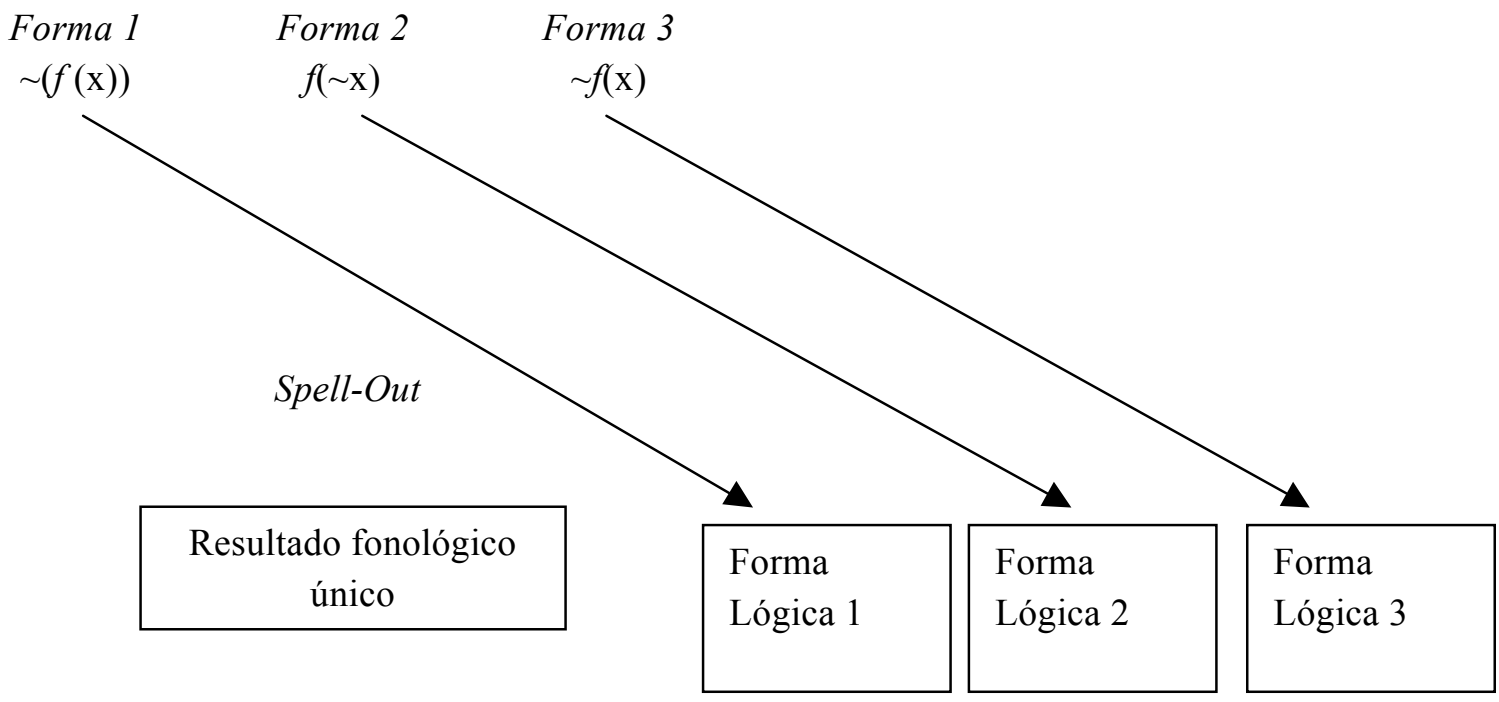

Vemos que tres representaciones semánticas que difieren en cuanto al alcance relativo del operador negativo pueden materializarse de manera idéntica, como ocurre por ejemplo en los casos del llamado "Neg-Raising" (Collins y Postal 2012) en lenguas como el inglés. El problema del alcance

\footnotetext{
${ }^{3}$ Crucialmente, distinguimos "necesidad" en un sentido lógico de "modalidad deóntica", en un sentido lingüístico. La necesidad lógica está relacionada con la satisfacción de una función en todos los mundos posibles (como el caso de los designadores rígidos en Kripke) o, más tradicionalmente, con la verdad a priori de una proposición en virtud de sus partes constitutivas (por ejemplo, una tautología).
} 
relativo de la modalización cuando se presentan casos con más de un modal, uno epistémico y uno deóntico es análogo: ¿puede inferirse la FL directamente a partir de la FF? Un sistema con interfaces transparentes está forzado a asumir que sí, pero, como veremos más abajo, no parece ser el caso. Por otro lado, modelos más complejos (la "arquitectura paralela" de Jackendoff 2002, por ejemplo) utilizan reglas de mapeo entre niveles (Estructura ConceptualEstructura Fonológica, en el caso que nos interesa), que si bien pueden redundar en mayor adecuación descriptiva, merman la elegancia teórica, al multiplicar los niveles en un movimiento teórico que no siempre está justificado por la "necesidad" en sentido técnico.

Habiendo revisado brevemente las concepciones más importantes dentro del ámbito de la Modalidad como categoría semántica, pasaremos ahora a revisar la interpretación semántica en los modelos generativos, para encaminarnos ya al planteo del problema de la introducción: ¿podemos subsumir el ascenso a una relación dictum-modus?

\section{INTERPRETACIÓN SEMÁNTICA EN EL MODELO GB: LA TEORÍA TEMÁTICA}

La teoría temática surge en el modelo generativista de Rección y Ligamiento (Chomsky 1981, 1986a, 1986b, Chomsky y Lasnik 1991), como una forma de incluir elementos de la interpretación semántica en la sintaxis. Como toda "subteoría" (también llamadas "módulos"), constaba de principios propios, condiciones de buena formación que se aplicaban a representaciones estructurales, de ahí el marcado sesgo representacionalista del modelo (en oposición al modelo más marcadamente derivacional de la década de los 70). Los niveles, Estructura-P, Estructura-S, Forma Fonética y Forma Lógica estaban sometidos a estas condiciones, y para que una expresión estuviera bien formada, debía cumplir las condiciones aplicadas a cada nivel. Las teorías se aplicaban a niveles determinados, de la siguiente forma:

- Estructura-P: Teoría Temática, Teoría de la X-barra, Principio de Proyección

- Estructura-S: Teoría del Caso, Teoría del Ligamiento, Principio de la Categoría Vacía, Teoría del Control, Condición de Subyacencia (Teoría del Alindamiento), Principio de Proyección

- FL: Teoría del Ligamiento, Principio de Proyección, Principio de la Categoría Vacía, Principio de Interpretación Plena (PIP)

- FF: PIP

El criterio temático es el principio fundamental de la teoría temática (o “teoría- $\left.\theta^{\prime \prime}\right)$, es una condición de buena formación que se aplica a la Estructura$\mathrm{P}$, estableciendo en su primera formulación una relación de uno-a-uno entre elementos y roles temáticos. Su formulación clásica, extraída de Chomsky (1981: 36) es la siguiente: 
Criterio temático: Cada argumento tiene uno y sólo un rol temático, y cada rol temático es asignado a uno y sólo a un argumento (traducción nuestra).

Teniendo en cuenta que un elemento puede tener más de un rol temático en su posición base, Chomsky reformula el criterio temático más adelante en su libro, añadiendo el corolario de que un elemento no puede adquirir roles temáticos en el curso de la derivación. Es decir, el movimiento a posiciones temáticamente marcadas queda vedado por principio.

\subsection{Verbos de ascenso y verbos de control}

El criterio temático tal como lo hemos formulado fue un elemento determinante en la diferencia entre construcciones de ascenso (raising) (c y d) y de control (a y b) (Polinsky 2009, Hornstein 2000, 2003), como las siguientes:

(6)

a) John wants/tries/hoped to finish the work.

b) Juan quiere/trata de/espera terminar el trabajo.

c) John seemed/appeared/was likely to finish the work.

d) Juan parecía terminar el trabajo

La diferencia entre los dos grupos de construcciones estaría dada por cuestiones de teoría temática. La posición de ensamble del sujeto del verbo de control (ejemplos a y b) está marcada temáticamente, mientras que la misma posición del verbo de ascenso no lo está. Esto equivale a decir que el verbo de ascenso, por no ser un asignador temático, no establece restricciones semánticas respecto del elemento que pueda ocupar la posición de sujeto de ese verbo. Si el verbo subordinado es también un asignador temático, entonces se presenta un problema: si [John] se origina en la cláusula subordinada, entonces habría un movimiento de posición temática a posición temática motivado por razones de Caso que violaría el criterio tal y como ha sido formulado. En el caso del verbo de ascenso, se da un movimiento de posición temática a posición no temática, ya que estos verbos no serían asignadores. El problema de los verbos de control y el criterio temático hizo necesaria la postulación de una categoría vacía $(\mathrm{CV})$ que ocupara la posición de sujeto en la cláusula subordinada y recibiera Caso y un rol temático dentro de esta cláusula, mientras que el sujeto realizado fonológicamente sería insertado directamente en la cláusula matriz, recibiendo rol del verbo de control. Esta CV sería PRO (Chomsky 1981, 1982). La estructuras de (a) y (c) a la luz de estos datos serían las siguientes (omitiendo el movimiento de Spec-VP a Spec-TP):

(7)

a') Johni wants/tries/hopes [PROi to finish the work]

$\Theta 1$

$\Theta 2$ 
$b^{\prime}$ ) Johni seemed/appeared/was likely [ti to finish the work]

$\Theta 1$

Si la E-P es la expresión pura de las relaciones temáticas por inserción léxica en diagramas arbóreos que cumplan con los requerimientos de binariedad (dos ramas por cada nodo no terminal) y endocentrismo (toda proyección tiene un núcleo) de la teoría de X-barra, entonces todas las posiciones- $\theta$ deben llenarse en ese nivel mediante la operación Satisfacer. Así, sería imposible que el sujeto de un verbo de ascenso se originara directamente en la cláusula matriz, porque habría una posición- $\theta$ sin llenar en la subordinada en E-P. A la inversa, el sujeto de un verbo de control no podría originarse en la cláusula subordinada, ya que una posición temática en la cláusula matriz quedaría vacante en E-P, y el movimiento entre posiciones temáticas está vedado. Esta distinción entre ascenso y control sería fundamental en las primeras teorías sobre modalidad en la sintaxis, ya que la distinción epistémico-deóntico se correspondía con ascenso-control, en propuestas como la de Ross (1969), quien considera que los modales deónticos asignan rol temático (como los verbos de control), mientras que los epistémicos, no (como los verbos de ascenso). Roberts (1985) y Zubizarrieta (1982) siguen la misma línea, dentro del marco GB. Esto implica que los deónticos, que se comportan como verbos (léxicos) de control, toman como complemento una cláusula no finita con un sujeto PRO (como en 7a), mientras que los modales epistémicos toman como complemento una cláusula no finita cuyo verbo léxico asigna rol temático al sujeto, pero no puede asignarle caso. De esta forma, el sujeto del infinitivo subordinado se mueve a la posición de especificador del modal, dejando atrás una huella. Esto implica, además, que los modales no serían auxiliares, sino, al menos en los casos paralelos al fenómeno de control, verbos léxicos, a insertarse en Estructura-P en VP antes que en TP (a donde pueden moverse dependiendo del seteo del parámetro pertinente en lenguas particulares).

Ya dentro del Programa Minimalista, Wurmbrand (1999) se opone a esta identificación, aduciendo que tanto los modales epistémicos como los deónticos son en realidad verbos de ascenso, por lo que el sujeto léxico siempre se origina dentro del ámbito del infinitivo subordinado. De tal forma, los modales se comportarían, independientemente de su semántica epistémica o deóntica, como verbos de ascenso. Así, las ambigüedades en alcance (scope) que se observan en los casos de ascenso se observarían también con verbos modales:

(8) Somebody from New York must have won in the lottery (Wurmbrand 1999: 607, ej. (16))

Wurmbrand (1999: 607) propone las siguientes lecturas para (8):

"a. In view of the evidence available it is necessarily the case that somebody from N.Y. won the lottery 
b. There is somebody from N.Y. and in view of the evidence available it is necessarily the case that he won the lottery."

En rigor de verdad, no es claro que las lecturas se desprendan exclusivamente de la posición del verbo en relación con el sujeto a nivel de FL. La presuposición [there is somebody from N.Y.] se genera independientemente por propiedades de las construcciones nominales (ver Strawson 1950 para discusión). Es decir, el cuantificador existencial que aparece en la descripción definida $\exists(\mathrm{x}) \mid$ humano(x) \& de N.Y.(x) - a grandes rasgos- interactúa con el resto de los elementos de la estructura sintáctica de una forma que no parece depender directamente de la categoría "verbo de ascenso", si es que tal categoría es aceptable en primer lugar (lo que cuestionaremos en secciones más abajo). Sí estamos de acuerdo con una propuesta de Wurmbrand, que consiste en sostener que los verbos modales no asignan rol temático al elemento que aparece en posición de sujeto, pero nuestro razonamiento, se verá, será inverso: no es el caso que los modales no asignen rol temático porque sean verbos de ascenso, sino que los verbos de ascenso no asignan rol temático a Spec-TP porque, precisamente, son un subtipo de verbos modales. Los modales pueden o no asignar un rol temático al elemento que aparece en posición de sujeto (es decir, materializado), pero siempre seleccionan un argumento externo semántico, el llamado "sujeto modal". La diferencia es sutil pero definitoria: la teoría de Wurmbrand predice que no hay modales que no sean verbos de ascenso, lo que requiere mantener las dos categorías teóricas, mientras que nosotros proponemos subsumir el fenómeno del ascenso (cuya naturaleza es todavía poco clara: ¿es semántica? ¿sintáctica?) al fenómeno semántico de modalidad, desde una perspectiva de interfaz.

\section{MODALIDAD EN LA SINTAXIS: DOMINIOS PROLÍFICOS Y DOMINIOS INFORMACIONALES}

En esta sección vamos a analizar cómo podemos representar la modalidad en la estructura sintáctica, de manera tal que la interpretación del enunciado (que consiste, desde una perspectiva pragmático-semántica, en la extracción de explicaturas e implicaturas, de acuerdo con Teoría de la Relevancia, ver Wilson y Sperber 2003 para discusión y detalles) esté, como ya hemos dicho, pre-subdeterminada por la sintaxis: no hay nada en la inferencia que no esté previsto por un elemento procedimental presente en la sintaxis, y no hay nada en la sintaxis que no sea funcional a la inferencia (una versión enriquecida del Principio de Interpretación Plena de Chomsky 1995).

Para representar la modalidad en la estructura sintáctica, debemos recordar que la modalidad modifica a un contenido proposicional, por lo que, en términos sintácticos, el nodo que codifique rasgos procedimentales de modalidad debe dominar a la proposición, tener alcance (scope) sobre ella. Consideramos, como es frecuente desde los estudios de Bally, que la modalidad 
es un functor que toma como argumento a la proposición. Antes de entrar en el análisis de una derivación, debemos introducir una herramienta teórica que nos será de gran utilidad: la hipótesis del Sintagma Tiempo escindido (Split-TP hypothesis). ${ }^{4}$ Esta herramienta teórica nos permitirá una descripción detallada de los rasgos temporales, aspectuales y modales de la cláusula al tiempo que se respeta el Principio de Interpretación Plena, ya que sostendremos que los rasgos T, Asp y Mod nuclean sus propias proyecciones sintácticas, las cuales son totalmente interpretables por las interfaces (especialmente FL, ya que estos rasgos son fundamentales en la construcción de la explicatura). La "escisión" de TP en nuestro modelo surge a partir del análisis de la data empírica y el encuentro con ejemplos como los siguientes:

(9)

a) La ballena era un mamífero (T: pasado. Mod: indicativo. Asp: imperfectivo) Interpretación genérica

b) La ballena embistió el barco (T: pasado. Mod: indicativo. Asp: perfectivo) Interpretación específica

c) Quien haya llegado tarde tendrá media falta (T: pasado. Mod: subjuntivo. Asp: perfectivo) Interpretación genérica (para todo $\mathrm{X}$, si $\mathrm{X}$ llegó tarde, $\mathrm{X}$ tendrá media falta)

d) Quien llegó tarde tendrá media falta (T: pasado. Mod: indicativo. Asp: perfectivo) Interpretación específica

Vemos en los ejemplos (a) a (d) que los rasgos de Asp y Mod, independientemente, pueden hacer que una determinada interpretación sea la más accesible para el módulo inferencial (que nosotros identificamos con el sistema extralingüístico C-I), por lo que no nos parece conveniente agrupar esos rasgos en el mismo nodo terminal a los fines del licenciamiento de dimensiones como definitud, aunque luego, por un proceso de fusión (Halle y Marantz 1993, Embick y Noyer 2004) puedan materializarse en un mismo ítem de vocabulario. Ya que la contribución de estos nodos a la explicatura está asociada pero es perfectamente distinguible, consideramos necesaria, para una mejor descripción y explicación de una serie de fenómenos (de los cuales el licenciamiento de definitud es sólo un ejemplo, como hemos analizado en Krivochen 2012), la proyección de estos rasgos con interpretación semántica en frases. ${ }^{5}$ Las relaciones de dominio entre ellos son especialmente pertinentes para nuestra

${ }^{4}$ No debe confundirse la propuesta de Split TP que manejamos aquí con los trabajos de Pollock (1989) y Chomsky (1989), así como van Gelderen (2012) para una propuesta reciente. Ver Krivochen (2012) y Krivochen y Kosta (2013) para una presentación de la hipótesis dentro del marco que aquí trabajamos y un análisis detallado de sus implicancias para la derivación sintáctica y la computación inferencial.

${ }^{5}$ Para una implementación posible de la mecánica formal que permite la proyección de rasgos en sintagmas, dentro de un marco ortodoxo, ver Varas San Vicente (2008). 
argumentación, ya que permiten retomar las propuestas de Bally y Benveniste (e incluso la lógica modal, ver sobre todo Kracht 1999 y Goldblatt 2006 para versiones actuales y formalizadas de los planteos modales) y aplicarlas en un terreno mucho más formal, con miras a la integración del conocimiento. A partir de la construcción paulatina de la derivación intentaremos justificar la construcción de la explicatura. Siguiendo el análisis de Rachmand (2008) y trabajos relacionados, proponemos que el dominio de SV (y proyecciones asociadas como $\mathrm{S} v$ ) denota un evento (más específicamente, una entidad eventiva), bien causado bien incausado, pero genérico, como un SN sin capa funcional D que le otorgue referencia. Los eventos, a nivel conceptual, son entidades que se extienden en un continuo temporal (así como las entidades sortales se extienden en espacio). $\mathrm{T}$ debe dominar a $\mathrm{V}$ porque es el equivalente de D, sitúa al evento "genérico" en un tiempo determinado respecto de un punto de referencia. En resumen, $\mathrm{D}$ es a $\mathrm{N}$ lo que $\mathrm{T}$ es a V. En cuanto a Asp, la categoría codifica una decisión del hablante, la de presentar el evento ya delimitado por $\mathrm{T}$ perfectiva o imperfectivamente, es decir, como un punto atómico en el continuum, o en desarrollo, atendiendo a su complejidad interna (ver Comrie 1976). Por lo tanto, debe tener alcance sobre aquello que modifica. La proyección sintáctica de las instrucciones procedimentales de Asp, entonces, dominan al evento genérico anclado en T. Mod, por último, es la expresión de la subjetividad del hablante respecto del contenido proposicional (AspP, que contiene a TP). Si, además, consideramos que parte de lo que se considera que está en la "periferia izquierda" (Top, Foc) está en realidad en SMod (como los llamados "disjuncts" por Quirk et al. 1985, que tienen alcance sobre toda la proposición, no solamente sobre el evento), este nodo debe tener alcance sobre la proposición. Mod es modalidad, no sólo indicativo, subjuntivo o imperativo (que son meramente expresiones morfológicas de la modalidad en el ámbito verbal, el llamado "modo", las cuales no tienen un correlato semántico unívoco, como argumentamos en la Figura 1) sino cualquier elemento que afecte la interpretación de la proposición por tener alcance sobre ella en la interfaz FL: sustantivos como "necesidad", "posibilidad"; adverbios como "posiblemente", "afortunadamente", "desgraciadamente" (ejemplos de "disjuncts"), adjetivos como "posible", o "verosímil". Cada nodo, además, licencia determinado tipo de adjuntos, que pueden moverse de una proyección a la otra (o ensamblarse secuencialmente en una y otra proyección, de acuerdo con una mecánica typetoken como la que proponemos en Krivochen 2013 y Krivochen y Kosta 2013) para tomar rasgos modales, aspectuales, etc. De este modo, la distribución de adverbios en la interfaz sintaxis-semántica, por ejemplo, no estaría determinada por una jerarquía de proyecciones funcionales pre-determinada. En una estructura sintáctica semánticamente motivada, toda posición $P$ debe estar requerida y licenciada por las características semánticas de un elemento $e$, de manera tal que: 
(10) $\forall(P), \exists(e) \mid e(P)$

Es decir, para toda posición debe existir un elemento tal que la requiera (por eso la notación predicado-argumento). La representación sintáctica final, de acuerdo con la derivación explicitada en los párrafos anteriores, sería algo como lo que sigue (tomando como válida la representación de Hale y Keyser 2002 y Mateu Fontanals 2002 para el dominio eventivo causado, Sv):

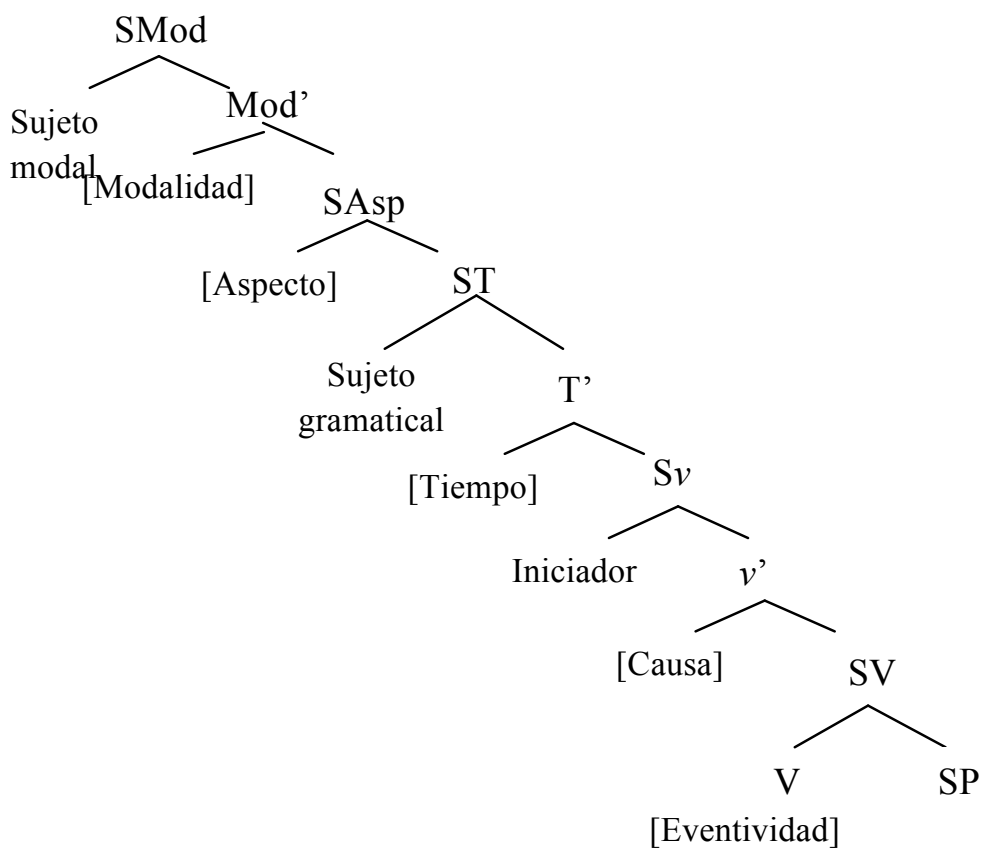

Desde luego, contra Cinque (1999) y trabajos relacionados, la jerarquía no se deriva de una estipulación apriorística respecto de la posición de un nodo $\alpha$ en un objeto sintáctico $\Sigma$ sino que (parafraseando el principio expuesto en (10), esencial para nuestro trabajo) para que $\alpha$ tenga una ocurrencia en $\Sigma$, esta ocurrencia debe estar requerido por la interfaz C-I y, a la vez, debe existir en $\Sigma$ un elemento que licencie a $\alpha$. Esto implica que un elemento aspectual (digamos, el adverbio "diariamente") no aparece dentro del dominio de Mod simplemente porque la contribución semántica de Mod no licencia un elemento aspectual. Así, garantizamos la ausencia de elementos superfluos en las derivaciones/ representaciones y la necesidad de recurrir a principios añadidos para justificar una estructura funcional extensa y fija, basada en el supuesto axiomático de la existencia de una Gramática Universal. Por lo dicho, no revisaremos la posición de Cinque (2006), ya que se inscribe en la misma línea, con la cual discrepamos en supuestos básicos.

Siguiendo con nuestra propuesta fuertemente componencial, los nodos no se interpretan aisladamente, sino que existen dominios informacionales (Krivochen 2011b, 2012), que, definidos contextualmente, proveen cierto tipo de información a las interfaces y resultan ser el locus en el que se dan las operaciones sintácticas como en una Configuración Mínima, al modo de los Dominios Prolíficos de Grohmann (2003, 2004). Así, podemos decir que el 
dominio locativo de $\mathrm{P}$ provee información espacial (literal o metafórica) y es el locus de licenciamiento de la interpretación de la esfera de caso del Dativo, el dominio de Sv, relacionado con la eventividad causada, es el locus de licenciamiento de la esfera casual (y temática) del Acusativo y la proyección de ST escindido (SMod, SAsp y ST) licencia la esfera del Nominativo; con lo que los casos quedarían reducidos a interpretaciones de relaciones locales entre elementos a nivel de FL. ${ }^{6}$ Un elemento puede tomar rasgos procedimentales de cada uno de estos núcleos mediante una operación estándar de Movimiento de Núcleo a Núcleo o, en nuestra propia propuesta (Krivochen 2013), a partir del ensamble de un token del elemento pertinente en cada posición pertinente a los fines semánticos; por lo que si un verbo manifiesta morfológicamente rasgos de eventividad causada (un transitivo o un inergativo, por ejemplo), además de Tiempo, Aspecto y Modo, estamos hablando de un movimiento en la sintaxis en sentido estricto y un proceso de fusión morfológica post-Materialización, al modo de Morfología Distribuida (Halle y Marantz 1993). Claro está, y este punto es fundamental para nuestra argumentación, que un ítem puede generarse independientemente en cualquiera de esos nodos, así, un auxiliar que manifieste rasgos exclusivamente aspectuales (por ejemplo, el [haber] perfectivo en español o su equivalente [have] en inglés), no habrá surgido en una capa léxica como SV, sino que será una materialización de los rasgos procedimentales del núcleo Asp y de Asp solamente. En el caso que nos interesa, debemos concentrarnos en la proyección de los rasgos modales, es decir, SMod.

El problema al que debemos enfrentarnos es establecer cuál es la composición del núcleo Mod en términos de rasgos, cuál es la instrucción procedimental asociada a ese nodo y en qué rasgos vamos a codificarla. Siguiendo a Leech (1971) y Palmer (1986), trabajaremos con la dimensión [realis] y dos valores,,.$+-^{7}$ Asumimos que todas las variedades de la modalidad son expresables a partir de estos valores, y los que no resultan inmediatamente computables, son resultado de un proceso inferencial, ya que Mod es una categoría procedimental. Esta caracterización nos ha ayudado (ver Krivochen 2012a) a establecer una caracterización de los "tiempos" en términos modales:

Supongamos que la distinción básica en términos temporales sea $[ \pm$ presente], es decir, simultáneo a la enunciación versus no simultáneo a la enunciación (o al punto de referencia que se haya fijado, en un sistema más complejo como el de Reichenbach). No obstante, esta distinción sola resulta

\footnotetext{
${ }^{6}$ Para un análisis detallado de la propuesta, ver Krivochen (2012).

7 Trabajaremos, por comodidad expositiva, con un sistema de rasgos estándar, como los define Juan Uriagereka en los comentarios a Derivation by Phase (Chomsky 1999). No obstante, la presente propuesta puede perfectamente adaptarse a un marco de Minimalismo Radical reemplazando rasgos binarios por dimensiones que, in abstracto, se expresen como una potencialidad continua, no como valores fijos y binarios.
} 
insuficiente, puesto que conlleva la indeseable consecuencia de agrupar pasado y futuro, siendo que mantienen notables diferencias semántico-pragmáticas entre sí.

A partir de un análisis de la lengua Dyirbal, Comrie (1985) sostiene que hay una distinción entre pasado-presente y futuro, caracterizable en términos de Modalidad. Es decir, pasado y presente son [+ realis], factuales; mientras que el futuro es [- realis], es decir, no factual. Esta distinción, a su vez, se combina con el rasgo de naturaleza temporal [ \pm presente], y con el Modo verbal, por lo que una proposición [- realis] puede expresarse en pasado ([- presente]), combinado con el modo subjuntivo, como en: "Si Juan estudiara más, le iría mejor en los exámenes". Presente, de acuerdo con nuestro planteo, se definiría entonces como [+ realis] [+ presente], pasado, como [+ realis] [- presente] y futuro, como [- realis], [- presente].

Ya que hemos distinguido diferentes dimensiones de la Modalidad, intentaremos dar cuenta de sus realizaciones sintácticas, diferenciando a su vez aquellas dimensiones que afectan a la proposición en tanto el hablante realiza una evaluación de algún tipo, que, como veremos, afectan a las explicaturas proposicionales en algunos casos (como los verbos de ascenso y los factivos) y aquellas dimensiones relacionadas con la "fuerza ilocucionaria", mejor expresada en términos de explicaturas de nivel superior. Las relaciones modales de Bally, junto con la modalidad de enunciado entran dentro del primer grupo. Las modalidades de enunciación y los procedimientos accesorios de Benveniste, forman parte del segundo grupo. La distinción es paralela a, y asimilable con, la distinción fregeana entre función y concepto, pero el desarrollo de tal postura requeriría más espacio del que disponemos aquí.

Debemos hacer una aclaración en este punto: no todos los usos de "parecer" están cubiertos por la etiqueta de "ascenso", y no todos han sido analizados dentro de los estudios gramaticales formales. A continuación, se analizan algunos usos de este verbo que no entran dentro de esta categoría: "Parecer" como una expresión de modalidad. Afirmamos aquí que "parecer" puede funcionar como una expresión de modalidad axiológica o epistémica, dependiendo del contexto. Por ejemplo:

i) ¿Te parece lo que hizo esta mina?

ii) ¿Te parece que tomar esto me va a hacer bien?

En el primer ejemplo, tenemos un valor axiológico claro. El significado es "te parece bien", con la implicatura conversacional que el hablante piensa claramente que lo que la mujer en cuestión hizo estuvo en efecto mal. En el segundo ejemplo, sin embargo, este análisis no daría resultado, ya que el significado que obtenemos no es "te parece bien" sino "te parece verdadero". La 
evaluación es, pues, en términos modales epistémicos. Los mismos efectos pueden ampliarse al [seem] inglés, incluso cambiando la fuerza ilocucionaria:

i) John seems to be a nice guy

ii) Does it seem OK to you to betray your best friend?

Nótese que (12 i) presenta un valor epistémico, es decir, el hablante presenta la proposición [John is a nice guy] como algo no seguro, susceptible de duda. La relativización del valor de verdad de la proposición se hace más evidente cuando se materializa el sujeto modal mediante un SP [to me], que es considerado en términos temáticos tradicionales un "experimentante" (Belleti y Rizzi 1988, Torrego 1996). En este caso, el supuesto "experimentante" no es sino el sujeto modal, el que realiza el juicio epistémico, disociado completamente del sujeto gramatical [John]. Por eso, las posiciones que hemos identificado en el árbol de (10) entre sujeto gramatical (licenciado como tema, en términos informacionales, por la posición externa de $\mathrm{T}$, como argumentamos en Krivochen y Kosta 2013) y sujeto modal, licenciado exclusivamente por el núcleo Mod. Veremos más abajo cómo estos dos "sujetos" pueden coincidir, lo que requiere un proceso de unificación en la interfaz semántica.

El segundo ejemplo contiene un elemento valorativo explícito, [OK], lo que facilita el análisis. No obstante, sería un error señalar que la lectura surge simplemente del elemento [OK]: si bien este proporciona información respecto a las características del juicio afectivo (recuperando la categoría de Maingueneau), no es el elemento modal en sí. De hecho, $[\mathrm{OK}]$ podría haber coocurrido con otros verbos sin generar una lectura modal:

(14) This shirt suits you OK

El problema, se ve, es más complejo de lo que parece. Sostenemos que, tanto en los casos españoles como en los ingleses, la lectura modal está dada por la presencia del verbo "de ascenso", categoría que revisaremos en la sección siguiente.

En nuestra conclusión se tratará de extender este análisis y explicitar las consecuencias que tendría para la sintaxis de los verbos de ascenso. Si tenemos éxito, esperamos estar en el camino de una teoría más simple y uniforme de los denominados "verbos de ascenso", la unificación de Movement y Construal en favor de Construal. ${ }^{8}$

8 Para la perspectiva opuesta, ver Grohmann (2003) y Hornstein (2003). Culicover y Jackendoff (2005, capítulo 12) defienden la idea tradicional de que el control no involucra movimiento de ningún tipo, más cercana a lo que proponemos aquí. 


\section{CONCLUSIÓN: HACIA UNA TEORÍA RADICALMENTE MINIMALISTA DEL "ASCENSO"}

En esta sección vamos a tomar todo lo que hemos dicho hasta ahora como válido (al menos en parte) en un cierto nivel de análisis, y trataremos de profundizar nuestra comprensión de la semántica y la sintaxis de los verbos como "parecer", el ejemplo paradigmático del fenómeno de ascenso a la posición de sujeto -especificador- de ST (raising-to-subject). A tal efecto, confrontaremos nuevamente ascenso y control, para ver en qué se diferencian desde una perspectiva de interfaz. La estructura (informal) lógica de una oración como (11 i) es (11 ii):

i) Yo quiero [ir]

ii) querer (yo, (ir (yo)))

Esto es, tenemos dos predicados diferentes y dos argumentos diferentes, uno por cada predicado. De este modo, tenemos también dos asignadores temáticos, como hemos visto más arriba. Resulta útil para el planteo del problema y la comprensión del fenómeno confrontar estos ejemplos con los siguientes:

i) Juan (me) parece trabajar

ii) * Parecer (Juan, (trabajar (Juan)))

¿Cuál es exactamente el problema con (16 ii)? La respuesta es simple, y ya conocida: [Juan] no es un argumento de "parecer", sino de "trabajar". ¿Qué está haciendo "parecer" ahí, entonces? Se trata, proponemos, de una expresión de modalidad, que indica la actitud del hablante con respecto al contenido proposicional en términos epistémicos. Es decir, [Juan trabaja] es una proposición a la que puede asignársele un valor veritativo, un dominio- $\theta$ completo (recordemos que el dominio $\phi$ se encuentra fuera de la proposición considerada estrictamente para poder licenciar rasgos en los argumentos ya que se encarga, de acuerdo con Grohmann 2003; de las propiedades de Agree). El hablante puede entonces elegir presentar esa proposición como algo de cuyo valor veritativo no está seguro, y ahora es cuando "parecer" entra en juego, como una materialización de los rasgos semánticos (procedimentales) de SMod. El único argumento real de "parecer", en términos semánticos/lógicos, sería el experimentante (esté o no realizado léxicamente), que es en realidad, como hemos visto, el sujeto modal (al cual hemos separado del sujeto gramatical, aunque puedan, como hemos dicho, coincidir). Si esto es así, entonces sería posible sostener que no hay dos cláusulas, sino sólo una, con un dominio- $\theta$ (eventivo) completo y un elemento modal en el dominio- $\Omega$ (si se considera, 
como nosotros hacemos por razones obvias, que SMod es parte de este Dominio Prolífico, la periferia izquierda de los enfoques cartográficos). Una teoría radicalmente minimalista sería aquella en la que no tenemos necesidad de postular la existencia de dos cláusulas en una construcción de ascenso típica, sino sólo de una, y la hipótesis del ST escindido es, consideramos, la herramienta perfecta para el trabajo. Una consecuencia teórica directa de lo que hemos dicho es que no todas las proyecciones asociadas a lo que se ha llamado $\mathrm{T}$ (ense) (o Inflexión) forman parte del dominio- $\phi$, ya que no hay rasgos de concordancia licenciados por el núcleo procedimental Mod en este caso. Sin embargo, como demostramos en Krivochen (2012), Mod participa en el proceso de licenciamiento de rasgos de definitud en los argumentos SD, que puede considerarse un proceso análogo a los de cotejo en el dominio- $\phi$, ya que la definitud de los argumentos es pertinente a la construcción de una forma proposicional completa en la interfaz con C-I (i.e., una explicatura en términos relevantistas). No vamos a profundizar en el tema por el momento, ya que el status de Mod dentro del marco de los Dominios Prolíficos rebasa los límites de este trabajo. En cuanto a la forma materializada final, el movimiento que posiciona a [Juan] antes de [parece] siguiendo el Axioma de Correspondencia Lineal (Kayne 1994) se puede atribuir a dos procesos:

a) Un movimiento en Forma Fonética con el fin de satisfacer algún requisito de antisimetría (à la Moro 2000).

b) Un ensamble externo de un token justificado en términos de dinámica tema-rema, siguiendo los lineamientos de Krivochen y Kosta (2013).

Hasta el momento, en teoría sintáctica minimalista se ha hecho uso de un "rasgo" [PPA] (Principio de Proyección Ampliado, según el cual toda cláusula debe tener un sujeto, ver Chomsky 1981) para justificar la posición del sujeto en la estructura linealizada final. No obstante, es claro que la introducción de un elemento con el solo fin de justificar un movimiento es estipulativa, y hay una vasta literatura referida al tema (Epstein y Seely 2002, Grohmann, Drury y Castillo 2000, Miechowicz-Mathiasen 2009 entre muchos otros) además de nuestra propia propuesta en Krivochen (2012) y Krivochen y Kosta (2013), por lo que se intentará justificar el movimiento por medio de condiciones de interfaz, particularmente la llamada "estructura informativa", cuya pertinencia ubicamos en el nivel de FL.

Para analizar este punto es necesario tomar en cuenta la interpretación semántica asociada a cada nodo procedimental, y los efectos que el movimiento a una posición externa a este nodo pueda tener. Los nodos más importantes a este respecto son $v, \mathrm{~T}$ y Mod: las asunciones de las que se partirá serán que $v$, que se interpreta como el primitivo semántico [causa], licencia una posición externa para un iniciador (agente o fuerza) del evento denotado por SV, Spec-T está asociado con el concepto informacional de tema y la posición de Spec-Mod 
está reservada para el sujeto modal, coincida o no con el elemento ensamblado en Spec-v, luego movido (o re-ensamblado) a Spec-T si es temático.

Obsérvense los siguientes ejemplos, en estructuras de ascenso con SP experimentante frontalizado:

(17) A mí (no a Pedro), Juani me parece [ti un buen pibe]

La posición del SP genera inferencialmente un entrañamiento, hay un valor contrastivo en la posición por encima del sujeto. Por el contrario, (b) no genera entrañamiento contrastivo alguno, y si se supone que [Juan] está en la posición Spec-T, entonces la afirmación de arriba tiene ahora peso empírico:

(18) ?! Juani (no Pedro) me parece [ti ser un buen pibe]

La posición que genera entrañamientos se encuentra por encima del dictum, en la periferia izquierda. En consonancia con Rizzi (1997), esta será identificada con STop. ¿Por qué no mover [Juan] directamente a Top en lugar de a T? Porque, si [Juan] es tema, informativamente hablando, entonces, siendo que Top domina indirectamente a $\mathrm{T}$, Spec- $\mathrm{T}$ actuaría como barrera por Minimalidad para el movimiento: interviene en la relación Top-asociado (SD) como una posición viable para el movimiento. Además, la explicatura no podría construirse completamente: una oración con sujeto frontalizado no puede ser tética, por lo que puede decirse que habría colapso a nivel de la interfaz semántica.

El movimiento de la posición de ensamble a la posición final podría estar dado exclusivamente por la necesidad de que el argumento pertinente pase por distintas posiciones, licenciadas por diferentes nodos procedimentales, para determinar una interpretación más rica a nivel de la explicatura, es decir, el movimiento está motivado por condiciones de interfaz de la instancia post-sintáctica de C-I (el módulo inferencial): si un SD se ensambla en Spec-v y luego se mueve a Spec-T, entonces no sólo es iniciador de un evento causado, sino también tema de la oración. Y si hay un segundo movimiento a Spec-Mod, entonces además es el sujeto modal, que evalúa el dictum sobre el que Modo tiene alcance. Es decir, el hecho de que el sujeto modal pueda coincidir con el gramatical es en nuestra propuesta una lectura que hace C-I de la estructura sintáctica, a partir de las ocurrencias de un SD en ciertas posiciones clave (Spec-T y Spec-Mod). En este punto, se presenta una pregunta: ¿es necesario el paso por Spec-Asp? No necesariamente. La posición de Spec-Asp no parece relacionada con un rasgo semántico determinado: si bien los rasgos de Aspo están relacionados con la elección del hablante de presentar el evento como un punto (perfectivamente) o desarrollándose en el tiempo, atendiendo a su complejidad interna (imperfectivamente), ni el tema ni el sujeto modal son responsables de esta elección, sino el sujeto hablante-locutor (en términos de Ducrot 1980). Por lo tanto, el paso por Spec-Asp no parece pertinente a los fines de extraer una explicatura 
completa. Dado que no asumimos el rasgo [PPA], que motiva el ensamble a la periferia de las Categorías Funcionales Centrales (v, T, C), al contrario que Chomsky (1998), entonces la posición de especificador no necesita ser proyectada en todas las categorías funcionales-procedimentales (siguiendo el principio explicitado en (10)), con la consiguiente simplificación de la estructura y la computación, dado que no hay elementos ni pasos superfluos en las representaciones y las derivaciones respectivamente.

El árbol correspondiente sería el siguiente, presentando sólo un esqueleto muy básico y provisional (sin contar ni problematizar movimientos de núcleo a núcleo ni la conflación que da origen al verbo inergativo trabajar, para lo cual recomendamos ver la propuesta de Hale y Keyser 2002) que el lector puede llenar con sus primitivos favoritos (para detalles sobre la naturaleza de la proyección espacial escindida, ver Svenonius 2007, Acedo-Matellán y Mateu Fontanals 2010, teniendo en cuenta que cada uno extrae diferentes conclusiones de la escisión de P):

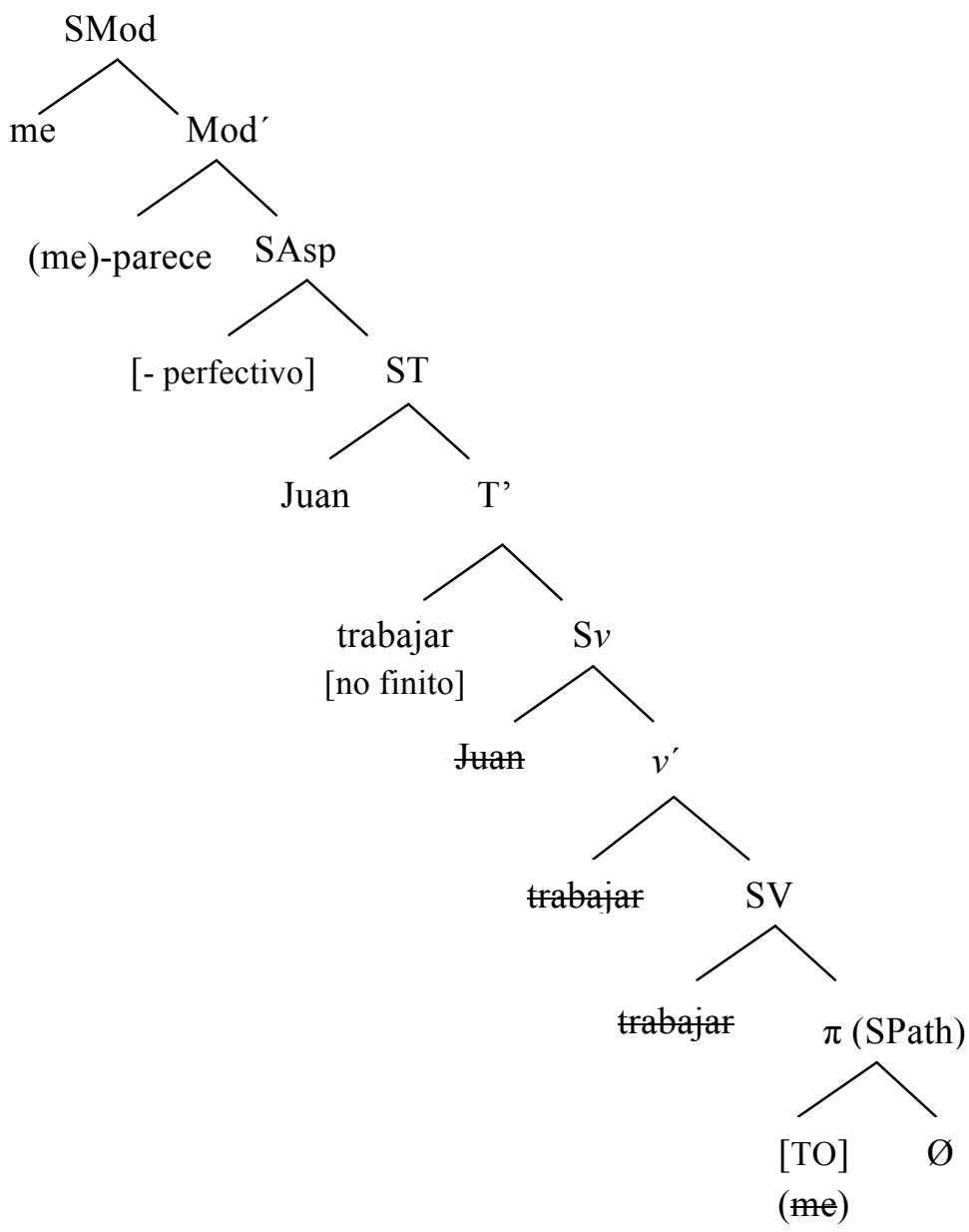

El sujeto modal es la entidad denotada por el clítico, [me]. El gramatical, por otro lado, es [Juan], lo cual puede leerse a partir de la estructura. Nuestra conclusión final sería que no hay ascenso en lo absoluto. Una estructura de frase 
semánticamente motivada permite dar cuenta del juego entre los dos sujetos sin necesidad de apelar a dos cláusulas o a la noción misma de "ascenso", que requiere estipulaciones respecto de las posiciones de los elementos en diferentes niveles de estructura relacionados mediante reglas, resultando inexorablemente en una teoría "poliestratal" (polystratal), o transformacional, contra lo cual se ha argumentado desde los 70 (Culicover y Jackendoff 2005: 16, ss.). Una teoría que trabaje solamente con ensamble externo de tokens en la estructura sintáctica es, consideramos, más económica que una que requiere dos tipos de filtros: unos para ensamble externo (desde el lexicón) y otros para ensamble interno (desde la misma estructura sintáctica).

\section{CONCLUSIÓN}

En este trabajo hemos realizado un reanálisis de las así llamadas "estructuras de ascenso" en la sintaxis generativa desde una persepectiva de interfaz entre la sintaxis y la semántica. Proponemos que cada cláusula tiene un SMod, en la que hay dimensiones procedimentales relacionadas con la modalidad, en términos de [ \pm realis] básicamente, ya sea materializadas (por ejemplo, como "parecer") o no, un caso que en general corresponde a la versión asertiva (a veces llamada "modalidad cero"), ya que es la opción no marcada: se presenta una propuesta y la ausencia de toda marca de modalidad se interpreta como "creo totalmente en lo que acabo de decir". Se trata de un compromiso con el contenido proposicional, que no debe confundirse con valor de verdad, siendo este último extralingüístico y carente de pertinencia a la sintaxis o al módulo inferencial. Es posible que los llamados "verbos factivos" (aquellos que generan una presuposición acerca del valor de la verdad del SC que seleccionan como complemento, como "lamentar", "creerse", etc.) también puedan ser analizados desde esta perspectiva, que nos da una herramienta útil para la descripción del comportamiento sintáctico-semántico de muchos verbos que han sido casi completamente ignorados hasta ahora en los estudios generativos ortodoxos, en contraste con la atención que se les ha prestado en los estudios de semántica.

\section{BIBLIOGRAFÍA}

ACEDo-Matellán, V. y MATEu FOnTANALS, J. (2010), From satellite-framed Latin to verbframed Romance: A syntactic approach, Universitat Autònoma de Barcelona, Departament de Filologia Catalana.

Ahern, A. y LeOnetTI, M. (2004), “The Spanish Subjunctive: Procedural Semantics and Pragmatic Inference", en Current Trends in the Pragmatics of Spanish, MárquezReiter y Placencia (eds.), Amsterdam, John Benjamins.

BALLy, C. (1947), El Lenguaje y la Vida, Buenos Aires, Losada.

BENVEnISTe, E. (1997), Problemas de Lingüística General I, México, Siglo XXI.

BosQue, I. y GutiÉRREZ REXACH, J. (2008), Fundamentos de sintaxis formal, Madrid, Akal.

CARSTON, R. (1998), The Relationship between Generative Grammar and (Relevance-theoretic)

Pragmatics, Ms. University College, London. 
CHOMSKY, N. (1999 [1965]), Aspectos de la teoría de la sintaxis, Barcelona, Gedisa.

CHOMSKY, N. (1980[1970]), "Sobre la nominalización", en Sintáctica y Semántica en la Gramática Generativa, Madrid, Siglo XXI, 25-75.

CHOMSKY, N. (1973), "Conditions on transformations", en Festschrift for Morris Halle, Anderson, S. y Kiparsky, P. (eds.), New York, Holt, Rinehart y Winston, 232-286.

CHOMSKY, N. (1981), Lectures on Government and Binding, Dordrecht, Foris.

CHOMSKY, N. (1988 [1982]), La nueva sintaxis. Teoría de la rección y el ligamiento, Barcelona, Paidós.

CHOMsky, N. (1986a), Barriers, Cambridge, Mass. MIT Press.

CHOMSKY, N. (1986b), El conocimiento del lenguaje, su naturaleza, origen y uso, Barcelona, Altaya.

CHOMSKY, N. (1995), The Minimalist Program, Cambridge, Mass., MIT press.

CHOMSKY, N. (1998), Minimalist Inquiries. The Framework, MIT Occasional Papers in Linguistics 15.

CHOMSKY, N. (1999), “Derivation by Phase” [Consulta: 20 de junio de 2013]. Disponible en: <http://www.letras.ufmg.br/fbonfim/Pdfs/Biblioteca\%20Virtual/Derivation\% 20by\%20Phase\%20-\%20Resenhado\%20por\%20Juan\%20Uriagereka\%202003.pdf>

CHOMSKY, N. y LASNIK, H. (1977), “Filters and Control”, Linguistic Inquiry (8): 425-504.

CHOMSKY, N. y LASNIK, H. (1991), “The Theory of Principles and Parameters", en Chomsky, N. (1995), Cap. 1, 13-128.

CINQue, G. (1999), Adverbs and Functional Heads: a Cross-Linguistic Perspective, Oxford, OUP.

CINQue, G. (2006), Restructuring and Functional Heads. The Cartography of Syntactic Structures, Vol. 4, Oxford, Oxford University Press.

Cinque, G. y RizzI, L. (2010), "The Cartography of Syntactic Structures", en The Oxford Handbook of Linguistic Analysis, Heine, B. y Narrog, H. (eds.), Oxford University Press, Oxford, New York, 51-65.

Collins, C. y POSTAL, P. (2012), Classical NEG-raising. Ms. [Consulta: 23 de junio de 2013]. Disponible en: <http://ling.auf.net/lingbuzz/001498>.

COMrIE, B. (1976), Aspect, Cambridge, CUP.

COMrIE, B. (1985), Tense, Cambridge, CUP.

Culicover, P. y JACKENDOFF, R. (2005), Simpler Syntax, Oxford, OUP.

Ducrot, O. (1980), Le Dire et le Dit, París, Minuit.

EGUREN, L. y FERNANDEZ SORIANO, O. (2004), Introducción a una sintaxis minimista, Madrid, Gredos.

EMBICK, D. y NOYER, R. (2004), Distributed Morphology and the Syntax-Morphology Interface, borrador del 25 de Octubre de 2004.

Epstein, S. y DANiel Seely, T. (2002), "Rule Applications as Cycles in a Level Free Syntax", en Derivation and Explanation in the Minimalist Program, Epstein, S.D. y Seeley, T.D. (eds.), Oxford, Blackwell, 65-89.

EsCANDELl Vidal, M. V. (2006), Introducción a la Pragmática, Barcelona, Ariel.

EsCANDELL VIDAL, M. V. y LEONETTI, M. (2000), “Categorías conceptuales y semántica procedimental", en Cien años de investigación semántica: de Michél Bréal a la actualidad. Tomo I, Madrid, Ediciones clásicas, 363-378.

García Negroni, M. M. y TORDEsillas ColAdo, M. (2001), La Enunciación en la Lengua, Madrid, Gredos. 
GoldBlatT, R. (2006), "Mathematical Modal Logic: a View of its Evolution", en Handbook of the History of Logic, Gabbay, D. M. y Woods, J. (eds.), Vol. 6. Elsevier BV.

GRICE, H. P. (1975), "Lógica y conversación”, en En búsqueda del significado, Valdés Villanueva, L. M. (Comp.), 1991, Madrid, Tecnos.

GrohmanN, K. K. (2003), Prolific Domains. On the Anti-Locality of Movement Dependencies, Amsterdam, John Benjamins.

GrohmanN, K. K., DRURY, J. y CARLOS CASTILlO, J. (2000), “No more EPP”, en WCCFL 19: Proceedings of the 19th West Coast Conference on Formal Linguistics, Billerey, R. y Lillehaugen, B. D. (eds.), Somerville, MA: Cascadilla Press, 153-66.

HALle, M. (1997), Distributed Morphology: Impoverishment and Fission, MIT Working papers in Linguistics 30.

HALle, M. y MARANTZ, A. (1993), "Distributed Morphology and the Pieces of Inflection", en The View from Building 20: Essays in Honor of Sylvain Bromberger, Hale, K. y Kayser, J., Cambridge, Mass., MIT press, 111-176.

HornsteIN, N. (1995), Logical Form: From GB to Minimalism, Oxford, Blackwell.

HornsteIn, N. (2000), Move! A Minimalist Theory of Construal, Oxford, Blackwell.

HORnSTEIN, N. (2003), “On Control”, en Minimalist Syntax, Hendrick, R. (ed.), London, Blackwell, 6-81.

HUANG, C. y JAMES, T. (1995), "Logical Form”, en Government and Binding Theory and the Minimalist Program, Webelhuth, G. (ed.), Oxford, Blackwell, 127-173.

KAYNE, R. (1994), The Antisymmetry of Syntax, Cambridge, Mass.: MIT press.

KRACHT, M. (1999), Tools and Techniques in Modal Logic, Studies in Logic and the Foundations of Mathematics No. 142, North Holland.

Krivochen, D. (2011a), "A New Perspective on Raising Verbs" [Consulta: 20 de junio de 2013]. Disponible en: <http://www.academia.edu/418711/_2011a_ A_New_Syntax_for_Raising_Verbs>

KRIVOCHEN, D. (2011b), “An Introduction to Radical Minimalism I: on Merge and Agree", en IBERIA, Vol. $3 \mathrm{n}^{\circ}$ 2, 20-62.

KRIVOCHEN, D. (2012), The Syntax and Semantics of the Nominal Construction, Potsdam Linguistic Investigations 8, Frankfurt am Main, Peter Lang Publishers.

KRIVOCHEN, D. (2013), Copies and Tokens: Displacement Revisited. Ms. UNLP. En revisión. [Consulta: 25 de octubre de 2013]. Disponible en: http://ling.auf.net/lingbuzz/001796

KRIVOCHEN, D. y KOSTA, P. (2013), Eliminating Empty Categories: a Radically Minimalist view on their Ontology and Justification, Potsdam Linguistic Investigations 11, Frankfurt am Main, Peter Lang Publishers.

LARSON, R. (1988), “On the Double Object Construction”, Linguistic Inquiry (19), 335391.

LEECH, G. (1971), Meaning and the English Verb, Longman.

LeONeTtI, M. y EsCANDEll VIDAL, M. V. (2004), “Semántica conceptual, semántica procedimental", en Actas del V congreso de Lingüística General, Madrid, Arco. 17271738.

Mateu Fontanals, J. (2002), Argument Structure. Relational Construal at the SyntaxSemantics Interface, PhD Dissertation, Bellaterra, UAB [Consulta: 20 de unio de 2013]. Disponible en: <http://www.tesisenxarxa.net/TDX-1021103-173806/>. 
MIECHOWICKZ-MATHIASEN, K. (2009), “There is no independent EPP in Slavic, there are only EPP-effects", en Studies in Formal Slavic Phonology, Morphology, Syntax, Semantics and Information Structure: Proceedings of FDSL-7, Leipzig 2007, Zybatow, G., Lenertova, D., Junghanns, U. y Biskup, P. (eds.), Frankfurt am Main, Peter Lang.

Moro, A. (2000), Dynamic Antisymmetry. Movement as a Symmetry Breaking Phenomenon. Cambridge, Mass., MIT Press.

PALMER, F. R. (1986), Mood and Modality, Cambridge, Mass., CUP.

POLINSKY, M. (2009), "Raising vs. Control”, Ms. Harvard University. [Consulta: 20 de junio 2013]. Disponible en: <http://scholar. harvard.edu/files/mpolinsky/files/raising_and_control.030311..pdf >

POSTAL, P. (1974), On raising. One rule of English grammar and its theoretical implications, Cambridge, Mass., MIT Press.

QUiRK, R., GREenBAuM, S., LEeCH, G. y SVARTVIK, J. (1985), A Comprehensive Grammar of the English Language, Longman.

RACHMAND, G. (2008), Verb meaning and the Lexicon, Cambridge, Mass., CUP.

RIZZI, L. (1997), "The Fine Structure of the Left Periphery", en Elements of Grammar, Haegeman, L. (ed.), Oxford, Blackwell.

RIzZI, L. (2004), "Locality and Left Periphery", en Structures and Beyond - The Cartography of Syntactic Structures, Vol 3., Belletti, A. (ed.), Oxford, OUP, 223-251.

SvenoniUs, P. (2007), "Projections of P", Ms. CASTL, University of Tromsø.

TORREGO, E. (1996), "Experiencers and Raising Verbs", en Current Issues in Comparative Grammar, Freidin, R. (ed.), Cambridge, Mass., MIT Press, 101-120.

WILSON, D. y SPERBER, D. (2003), “La teoría de la Relevancia”, en Revista de Investigación Lingüística, Vol. VII-2004, 237-286.

VAN GELDEREN, E. (2012), Clause Structure, Ms. Arizona State University.

VARAS SAN VICENTE, M. Á. (2008), “Categorías funcionales, categorías léxicas y rasgos. Un intento de simplificación”, en DICENDA, Cuadernos de Filología Hispánica, vol. 26.

WURMBRAND, S. (1999), "Modal verbs must be raising verbs", en Proceedings of the 18th West Coast Conference on Formal Linguistics (WCCFL 18), Bird, S., Carnie, A., Haugen, J. D. y Norquest, P. (eds.), Somerville, Mass, Cascadilla Press, 599-612.

YUS, F. (2010), "Relevance Theory", en The Oxford Handbook of Linguistic Analysis, Heine, B. y Narrog, H. (eds.), Oxford, Oxford University Press, 679-701. 